\title{
Prótesis metálicas para la reparación de cabezas de vigas de madera degradadas
}

\author{
Steel profiles for repairing deteriorated timber beam ends
}

$\underline{\text { C. González-Bravo }}^{(*)}$, F. Arriaga ${ }^{(* *)}$, G. Íñiguez-González ${ }^{(* *)}$, L. Maldonado ${ }^{(* *)}$

\section{RESUMEN}

Se describe un procedimiento para la consolidación de viguetas de forjado de madera con deterioro en las cabezas mediante perfiles de acero conectados a la madera desde la cara superior del forjado. La pieza de refuerzo es un perfil en $U$ de acero S275 conformado en frío con pletinas soldadas insertadas en la madera y conectada mediante tirafondos.

Se ensayaron 30 piezas a flexión obteniendo la rigidez y la capacidad de carga. Las probetas se dividieron en tres grupos. El primero compuesto por 10 piezas de madera laminada encolada de abeto con una sección de 180 x $200 \mathrm{~mm}$ y una longitud de $4.000 \mathrm{~mm}$; el segundo consistía en 10 piezas de madera aserrada de pino silvestre con la misma sección y longitud y, el tercero, estaba formado por otras 10 piezas de madera del género Pinus con una sección de 130 x 150 mm y 3.000 $\mathrm{mm}$ de longitud, procedentes de un edificio de Madrid con 120 años de antigüedad. Cada grupo de 10 piezas se dividió a su vez en dos grupos de 5 piezas. El primer subgrupo estaba formado por las piezas completas de madera y constituía el grupo de referencia. Las piezas del segundo subgrupo tenían una longitud inferior que se salvaba con una extensión del refuerzo metálico.

Los resultados indican que el sistema de refuerzo metálico permite resolver los problemas de falta de apoyo de la vigueta por deterioro de la madera que afecte en una longitud limitada (aproximadamente entre el 10 y el 20\% de la longitud).

853-5

Palabras clave: madera, acero, método de los elementos finitos, refuerzo, rehabilitación

\section{SUMMARY}

This paper describes the design of a procedure for the upgrading of timber floor joists with decayed ends using steel profiles connected to timber from the upper side of the flooring. The reinforcement consists on a steel S275 U cold formed shape with a plate inserted into timber and connected by means of screwing into the wood.

For the experimental verification 30 beams were tested in bending obtaining the stiffness and load carrying capacity. The specimens were divided into three groups. The first was composed by 10 pieces of glued laminated timber of Spruce with $180 \times 200 \mathrm{~mm}$ cross section; the second one was composed by 10 pieces of sawn timber of Scots pine with the same cross section; and the third one was constituted by 10 solid timber beams of genus Pine with $130 \times 150 \mathrm{~mm}$ cross section, from a 120 years old building in Madrid. Each group of 10 pieces was divided in two groups of 5 beams. The first sub-group was constituted by the beams with the whole length as a reference group. The pieces of the second one had a shorter length that was substituted by the steel extension.

It can be concluded that the steel profile reinforcement can solve the problems derivates from the unsafe support caused by wooden decay affecting a certain and limited length (approximately from 10 to $20 \%$ of the length).

Keywords: timber, steel, finite elements method, upgrading, rehabilitation 


\section{INTRODUCCIÓN}

Uno de los problemas más frecuentes en edificaciones con forjados de madera es la degradación de las cabezas de las viguetas en las zonas de apoyo. Este problema se presenta en las zonas húmedas como pueden ser los espacios destinados a servicio (baños y cocinas) y las zonas de apoyo en muros de fachada principalmente en las zonas donde existen repisas de balcones o molduras en la fachada. En estas situaciones la fábrica se humedecerá y la madera por higroscopicidad aumentará su contenido de humedad por encima del $20 \%$, lo que permite y desencadena un proceso de pudrición por hongos. También facilita el ataque de otros organismos xilófagos como es el caso de los anóbidos, insectos de ciclo larvario que muchas veces acompañan a los hongos de pudrición. Los daños afectan generalmente sólo al extremo de la pieza pero disminuyen y en la mayoría de los casos anulan su estabilidad y capacidad estructural, al desaparecer el apoyo en el extremo. El fallo final se suele producir cuando son varias las cabezas afectadas produciéndose la caída de una franja de forjado de entre 3

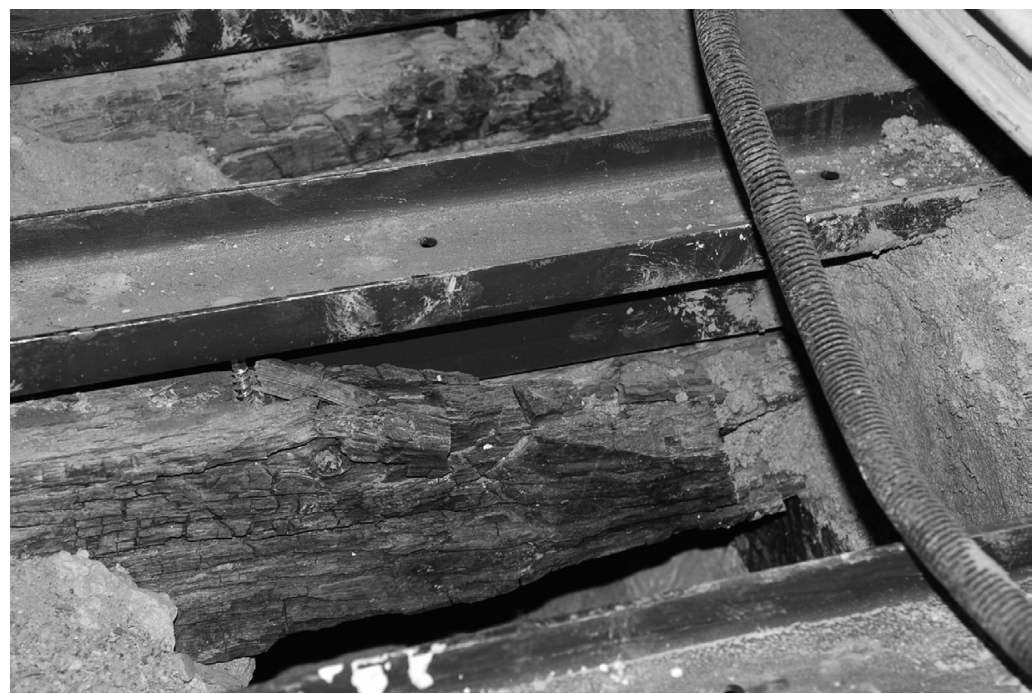

1. Prótesis en la cabeza de viguetas de forjado con daños de pudrición. ó 5 viguetas, aproximadamente, según sea la entidad de los daños.

En la actualidad, se emplean diversas soluciones para resolver este problema tan frecuente en las intervenciones de restauración y rehabilitación, que pueden agruparse en los cinco procedimientos siguientes. El primero consiste en la sustitución de las piezas afectadas preferiblemente por otras piezas de madera. Es la solución más simple y más conservadora con respecto a la integridad material de la estructura. El segundo caso corresponde a las soluciones de apeo desde la cara inferior con carácter permanente. Es el caso de la colocación de perfiles metálicos constituyendo una nueva línea de apoyo para las cabezas de las vigas (1). El tercero consiste en la reconstrucción de la parte dañada de la madera utilizando bien madera, generalmente en forma de láminas que se encolan a la pieza original $(2,3)$ o bien utilizando morteros epoxi que se conectan a la madera mediante barras generalmente de materiales compuestos (4, 5). El cuarto grupo está constituido por aqueIlas soluciones que emplean el acero para recuperar la longitud perdida de la pieza. Generalmente son soluciones muy simples y de baja eficacia práctica que consisten en la conexión a la madera sana de perfiles en forma de $L$ o de $U$ que sustituyen al tramo degradado de la pieza (1). Todas estas soluciones implican de forma casi inevitable la actuación desde la cara inferior del forjado, no sólo de apeo sino también de trabajo de intervención.

La posibilidad del refuerzo de vigas de madera con perfiles metálicos actuando exclusivamente desde la cara superior ha sido estudiada en trabajos anteriores por este equipo de investigación (6-8). En estos trabajos se estudió el refuerzo de vigas de madera mediante la colocación de unos perfiles de acero en la cara superior que se conectaban a la madera mediante tirafondos. La capacidad de carga de la viga se veía incrementada con el refuerzo y principalmente se aumentaba su rigidez. El empleo de este procedimiento permite la actuación desde la cara superior aunque es inevitable, en algunos casos, por razones de seguridad intervenir desde abajo para la colocación de los apeos mínimos necesarios o, en su caso, sistemas de apeo desde la cara superior.

El objetivo del presente estudio es analizar si la solución de refuerzo comentada puede también llegar a resolver de una manera simple un problema de deterioro y degradación en la cabeza de la viga. Hay que tener en cuenta que, por lo general, cuando existe la necesidad de incrementar la capacidad de carga de un forjado es normal que también existan problemas de deterioro de las cabezas de apoyo de las viguetas en las zonas húmedas.

En estudios anteriores $(6,8)$ ha sido comprobada la eficacia de este tipo de refuerzos actuando por la cara superior, dado que permite un trabajo de recuperación de la estructura de madera, sin necesidad de intervención por las dos caras del forjado, la demolición del entrevigado o la necesidad de apeo de la estructura. Por otro lado, el sistema de prótesis metálica permite, al igual que la solución de refuerzo mencionada, una homogenización del comportamiento del forjado tras la colocación de la losa superior del mismo, Figura 1. 


\section{MATERIALES}

El material de ensayo estaba formado por un conjunto de 30 piezas de madera divididas en tres grupos: 10 piezas de madera laminada encolada de abeto (Picea abies L. Karst) con una sección transversal de $180 \times 200 \mathrm{~mm}$ y una longitud de $4.000 \mathrm{~mm}$; 10 piezas de madera aserrada de pino silvestre (Pinus sylvestris L.) con igual sección y longitud y 10 piezas procedentes de un derribo de edificio con antigüedad aproximada de 120 años de madera del género Pinus, con una sección transversal media de $130 \times 150 \mathrm{~mm}$ y una longitud de $3.000 \mathrm{~mm}$.

Cada grupo de piezas de un material se dividió en dos subgrupos: el primero (subgrupo A) estaba constituido por 5 de las 10 piezas, elegidas al azar, y con la longitud completa. El segundo (subgrupo B) estaba formado por las 5 piezas restantes a las que se había reducido su longitud en una cantidad de 1,14 $\mathrm{m}$ para las secciones grandes $(180 \times 200 \mathrm{~mm})$ y 0,86 $\mathrm{m}$ para las piezas de menor escuadría de madera antigua. En las piezas de este subgrupo B el tramo de la pieza cortado (denominado en adelante zona de extensión) es sustituido por un perfil metálico según se describe a continuación (Figura 2).

Los perfiles de refuerzo fueron fabricados con chapa plegada en forma de $U$ de 4 y $5 \mathrm{~mm}$ de espesor con acero de calidad S275J0 según norma UNE-EN 10025 (9). Las chapas de acero fueron cortadas y taladradas mediante arco de plasma. Esta técnica resulta económica y muy adecuada al dejar las aristas limpias sin rebabas y con un cierto bisel lo que facilita su manipulación en obra.

Para las piezas con sección transversal de $180 \times 200$ mm (madera laminada y madera aserrada) se utilizó un perfil en $U$ (de $5 \mathrm{~mm}$ de espesor) con dos pletinas en la cara inferior (de $4 \mathrm{~mm}$ de espesor). Este perfil de refuerzo cambia en la zona de extensión sustituyendo las pletinas por un tubo rectangular hueco de 90,5 × 0,4 mm (Figura 3). Para las piezas de madera antigua que tenían una sección más reducida con dimensiones medias de $130 \times 150 \mathrm{~mm}$, se utilizó un perfil en $U$ (de $4 \mathrm{~mm}$ de espesor) con una sola pletina en la cara inferior (de $4 \mathrm{~mm}$ de espesor). Y de forma similar al caso anterior, en la zona de extensión, la pletina era sustituida por un tubo rectangular hueco de 60,4 × $0.4 \mathrm{~mm}$ (Figura 3). Las pletinas y los tubos estaban soldadas mediante arco de hilo continuo con cordones en cada lado, con una longitud de $30 \mathrm{~mm}$ y una separación de $300 \mathrm{~mm}$. En la Figura 3 puede observarse el detalle de la transición entre las pletinas y los tubos. En la Tabla 1 se recogen los valores estáticos de las secciones metálicas.
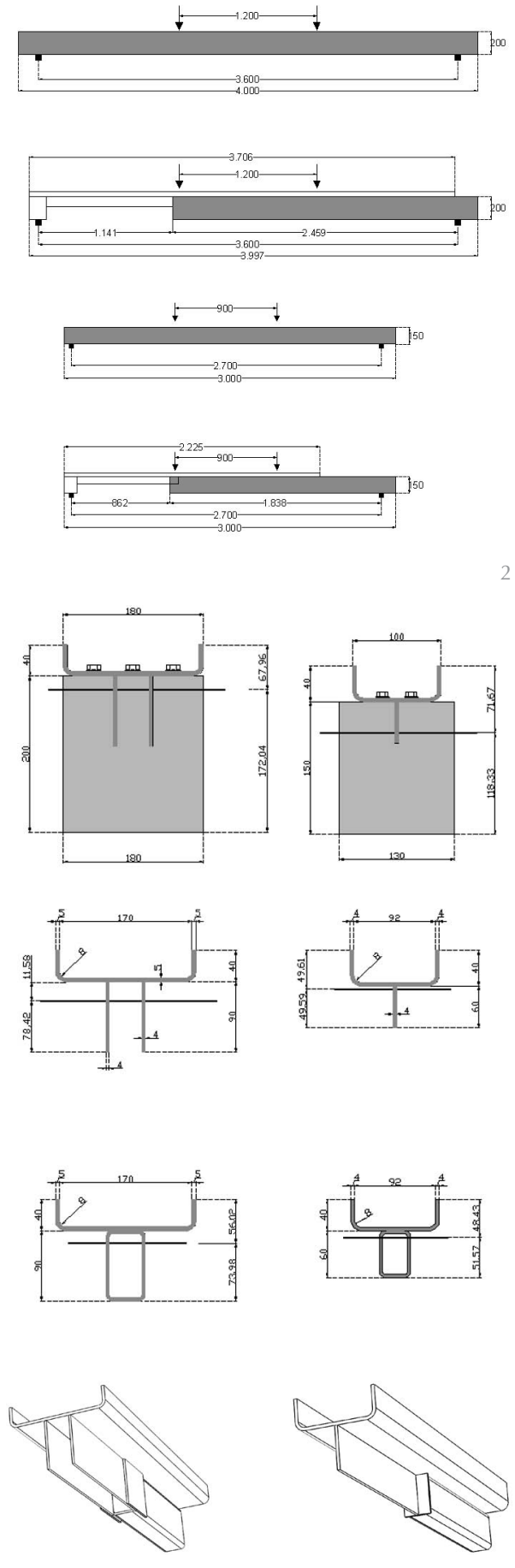

Como elementos de fijación se utilizaron tirafondos de $8 \mathrm{~mm}$ de diámetro con longitud de $90 \mathrm{~mm}$ para las secciones de $180 \times 200 \mathrm{~mm}$ y de $7 \mathrm{~mm}$ de diámetro con longitud de $70 \mathrm{~mm}$ para las secciones de 130 × 150 mm, según la norma DIN 571 (10). La fijación del refuerzo en las secciones de $180 \times 200 \mathrm{~mm}$ se realizó mediante la disposición de 3 filas de tirafondos al tresbolillo con una separación en la dirección paralela a la fibra de $100 \mathrm{~mm}$. Para las secciones de madera antigua, de $130 \times 150$ $\mathrm{mm}$ se utilizaron 2 filas al tresbolillo con una separación en dirección paralela a la fibra de 100 mm (Figura 4). El agujero en las chapas tenía un diámetro de $10 \mathrm{~mm}$ para el caso de
2. Arriba, las piezas de $180 \times 200 \mathrm{~mm}$ (pieza completa de madera y la pieza con la zona sustituida por perfil metálico); abajo, las piezas de $130 \times 150 \mathrm{~mm}$ (con la misma disposición).

3. Arriba: Refuerzo con perfil de acero en secciones de $180 \times 200 \mathrm{~mm}$ y de $130 \times 150 \mathrm{~mm}$.

Centro: Perfiles de refuerzo y de extensión y centros de gravedad. Abajo: Detalle de la unión entre pletinas y tubos. 
Tabla 1

Valores estáticos de las secciones metálicas

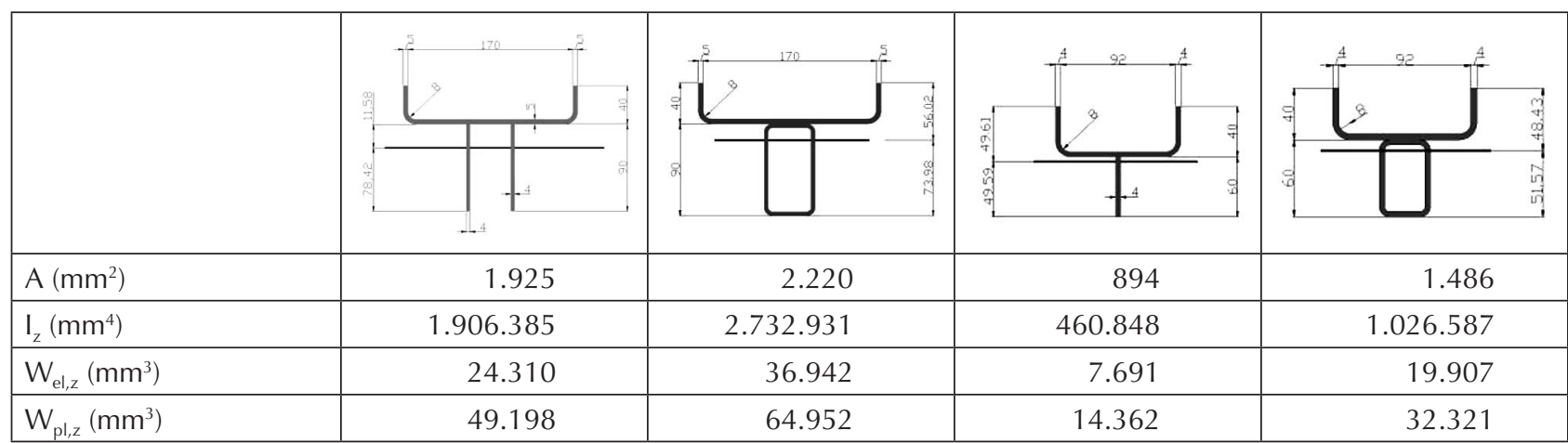
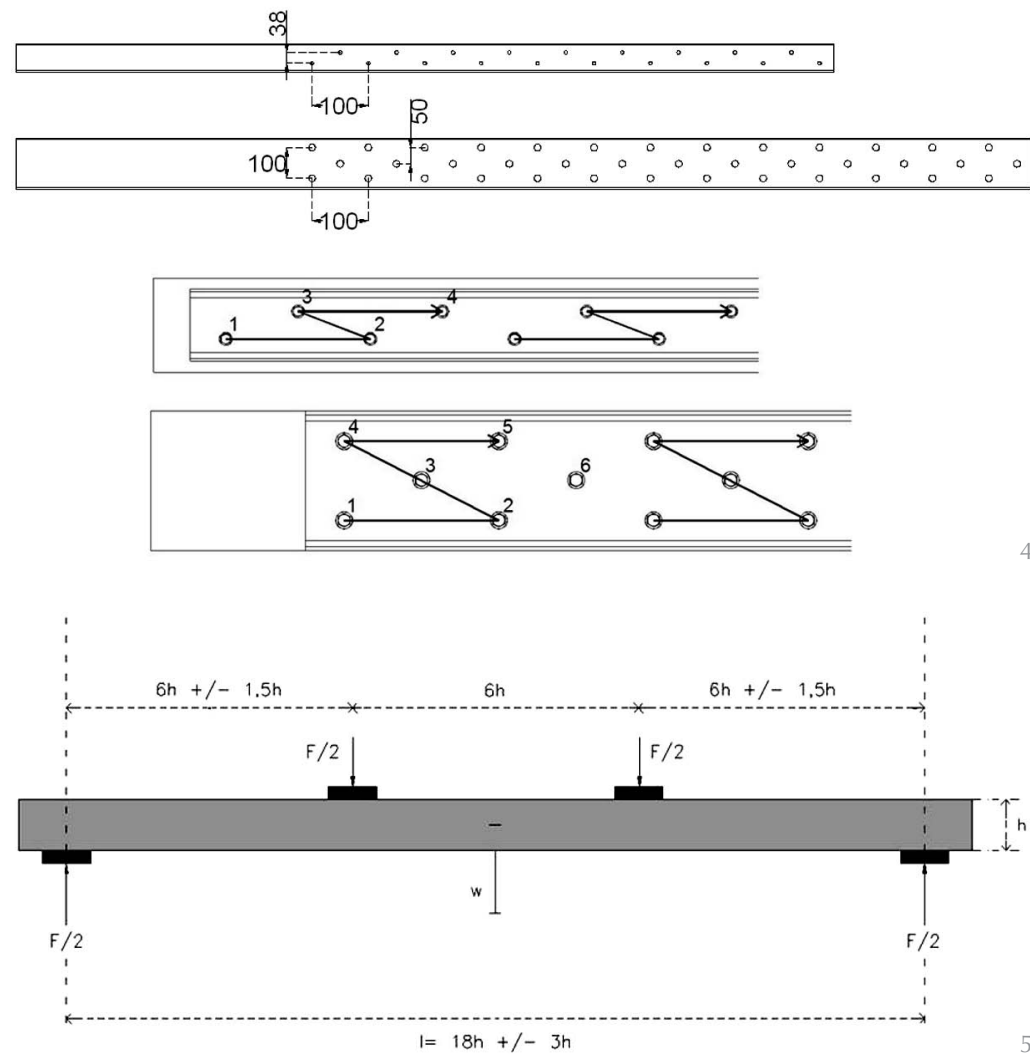

4. Arriba: Disposición de los tirafondos. Abajo: Secuencia de apriete de los tirafondos.

Disposición del ensayo de flexión.

los tirafondos de $8 \mathrm{~mm}$ y de $9 \mathrm{~mm}$ para el caso de los tirafondos de $7 \mathrm{~mm}$. Para la introducción de los tirafondos no se realizó un taladro previo debido a que se observó que no existía lograr un acercamiento entre la madera y el acero más gradual, (Figura 4).

\section{MÉTODO EXPERIMENTAL}

En primer lugar se obtuvo el módulo de elasticidad global en el ensayo de flexión según la norma UNE-EN 408 (11) de todas las piezas (Figura 5). De acuerdo con esta norma la luz (distancia entre apoyos) debe ser igual a 18 veces el canto de la pieza. Para las piezas de madera laminada y madera aserrada con una sección de 180 × 200 mm resultaba una luz de 3,6 m. En el caso de la madera antigua la sección variaba para cada pieza, por lo que se tomó una sección de referencia de $130 \times 150 \mathrm{~mm}$, resultando una luz de 2,7 m.

En la Tabla 2 se muestran los valores medios del módulo de elasticidad para cada material. Se observa como la madera laminada encolada tiene el mayor valor del módulo de elasticidad y el menor coeficiente de variación (5\%); y la madera antigua, presenta el módulo de elasticidad menor y con el mayor coeficiente de variación (28\%). De esta forma, se pueden analizar los resultados con mayor precisión en el caso de la madera laminada, pero también es posible acercarse a los resultados prácticos con materiales más variables como la madera aserrada y antigua. problema de fendado en la madera. El apriete se efectuó inicialmente de forma mecánica, pero el apriete final se realizó con una llave manual siguiendo un orden en zig-zag para
El contenido de humedad de las piezas se evaluó mediante xilohigrómetro de resistencia eléctrica de acuerdo con el procedimiento definido en la norma UNE-EN 13183-2 (12). El valor medio era de $13,4 \%$ para la madera laminada, 15,9\% para la madera aserrada y $11,0 \%$ para la madera antigua.

Tabla 2

Valor medio del módulo de elasticidad

y coeficiente de variación de los tres materiales ensayados

\begin{tabular}{|l|c|c|c|}
\hline Material & $\begin{array}{c}\text { Número } \\
\text { de piezas }\end{array}$ & $\begin{array}{c}\text { Módulo de elasticidad } \\
\left(\mathrm{N} / \mathrm{mm}^{2}\right)\end{array}$ & $\begin{array}{c}\text { Coeficiente de } \\
\text { variación (\%) }\end{array}$ \\
\hline Madera laminada & 10 & 10.999 & 5 \\
\hline Madera aserrada & 10 & 7.901 & 9 \\
\hline Madera antigua & 10 & 6.778 & 28 \\
\hline
\end{tabular}


Tabla 3

Módulo de elasticidad, tensión y momento de rotura a flexión de las piezas de los grupos A (testigo)

\begin{tabular}{|c|c|c|c|c|}
\hline Material & $\begin{array}{l}\text { Sección } \\
\mathrm{b} \times \mathrm{h} \\
(\mathrm{mm})\end{array}$ & $\begin{array}{l}\text { Módulo de elasticidad } \\
\qquad\left(\mathrm{N} / \mathrm{mm}^{2}\right)\end{array}$ & $\begin{array}{c}\text { Tensión de rotura } \\
\qquad\left(\mathrm{N} / \mathrm{mm}^{2}\right)\end{array}$ & $\begin{array}{l}\text { Momento } \\
\text { de rotura } \mathrm{M}_{\text {rup }} \\
(\mathrm{kN} \cdot \mathrm{m})\end{array}$ \\
\hline $\begin{array}{l}\text { Madera } \\
\text { laminada }\end{array}$ & $180 \times 200$ & $\begin{array}{r}10.916 \\
10.095 \\
9.634 \\
11.437 \\
11.122 \\
\end{array}$ & $\begin{array}{l}40,5 \\
29,6 \\
33,9 \\
41,3 \\
47,7\end{array}$ & $\begin{array}{l}48,570 \\
35,574 \\
40,722 \\
49,590 \\
57,216\end{array}$ \\
\hline \multicolumn{2}{|c|}{$\begin{array}{l}\text { Valor medio } \\
\text { Coef. de variación (\%) }\end{array}$} & $\begin{array}{r}10.641 \\
7,0\end{array}$ & $\begin{array}{l}38,6 \\
18,1\end{array}$ & $\begin{array}{r}46,334 \\
18,1 \\
\end{array}$ \\
\hline $\begin{array}{l}\text { Madera } \\
\text { aserrada }\end{array}$ & $180 \times 200$ & $\begin{array}{l}7.003 \\
8.317 \\
6.869 \\
8.214 \\
9.210 \\
\end{array}$ & $\begin{array}{l}23,2 \\
33,0 \\
25,2 \\
31,0 \\
42,3\end{array}$ & $\begin{array}{l}27,840 \\
39,552 \\
30,276 \\
37,164 \\
50,796\end{array}$ \\
\hline \multicolumn{2}{|c|}{$\begin{array}{l}\text { Valor medio } \\
\text { Coef. de variación (\%) }\end{array}$} & $\begin{array}{r}7.923 \\
12,4\end{array}$ & $\begin{array}{l}30,9 \\
24,3\end{array}$ & $\begin{array}{r}37,126 \\
24,3\end{array}$ \\
\hline $\begin{array}{l}\text { Madera } \\
\text { antigua }\end{array}$ & $\begin{array}{l}142 \times 156 \\
141 \times 165 \\
122 \times 156 \\
149 \times 169 \\
132 \times 151\end{array}$ & $\begin{array}{r}4.219 \\
7.225 \\
7.113 \\
7.580 \\
10.521\end{array}$ & $\begin{array}{l}10,1 \\
25,9 \\
23,6 \\
24,5 \\
39,0\end{array}$ & $\begin{array}{r}5,805 \\
16,542 \\
11,700 \\
17,348 \\
19,575\end{array}$ \\
\hline \multicolumn{2}{|c|}{$\begin{array}{l}\text { Valor medio } \\
\text { Coef. de variación }(\%)\end{array}$} & $\begin{array}{r}7.332 \\
30,5\end{array}$ & $\begin{array}{l}24,6 \\
41,7\end{array}$ & $\begin{array}{r}14,194 \\
38,7\end{array}$ \\
\hline
\end{tabular}

Las piezas de los grupos A fueron ensayadas a flexión de acuerdo con la norma UNE-EN 408 determinando de nuevo el módulo de elasticidad global, y la tensión y momento de rotura a flexión. En la Tabla 3 se muestran los resultados obtenidos.

Las piezas de los grupos B fueron ensayadas a flexión según la norma UNE-EN 408 para la determinación de la rigidez a flexión inicial $(E \cdot I)_{\text {in }}$. Posteriormente, se procedió al corte del tramo denominado de extensión y se colocaron los perfiles metálicos definidos en el apartado de Materiales para recuperar la longitud total de la pieza. Después se ensayaron en flexión según la misma norma y con la misma luz entre apoyos, Figura 6, determinando la rigidez a flexión eficaz de la pieza reforzada $(E \cdot I)_{\text {ef }}$ Y el momento de rotura en flexión $\left(M_{\text {rup }}\right)$. En la Tabla 4 se muestran los resultados obtenidos.

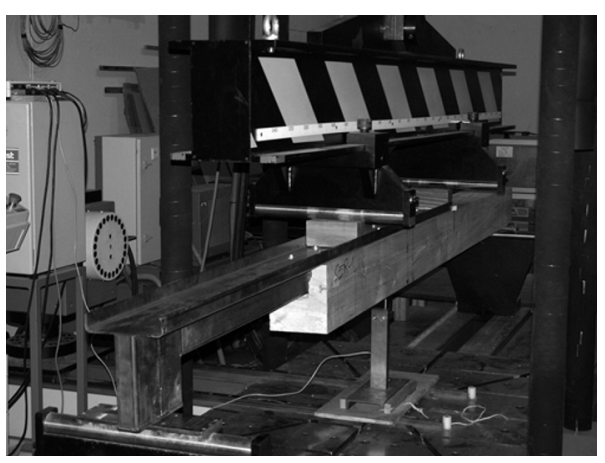

\section{Tabla 4}

Rigidez a flexión inicial y eficaz (con la extensión metálica) y momento de rotura a flexión de las piezas de los grupos B

\begin{tabular}{|c|c|c|c|c|c|}
\hline \multirow{2}{*}{ Material } & \multirow{2}{*}{ Pieza } & \multirow{2}{*}{$\begin{array}{l}\text { Sección } \\
b \times h(m m)\end{array}$} & \multicolumn{2}{|c|}{$\begin{array}{c}\text { Rigidez a flexión } \\
\left(\mathrm{kN} \cdot \mathrm{m}^{2}\right)\end{array}$} & \multirow{2}{*}{$\begin{array}{c}\text { Momento } \\
\text { de rotura } \\
M_{\text {rup }} \\
(\mathrm{kN} \cdot \mathrm{m})\end{array}$} \\
\hline & & & $\begin{array}{c}\text { Inicial } \\
(E \cdot I)_{\text {in }}\end{array}$ & $\begin{array}{c}\text { Reforzada } \\
(\mathrm{E} \cdot \mathrm{I})_{\mathrm{ef}}\end{array}$ & \\
\hline $\begin{array}{l}\text { Madera } \\
\text { laminada }\end{array}$ & $\begin{array}{l}1 \\
2 \\
3 \\
4 \\
5\end{array}$ & $180 \times 200$ & $\begin{array}{l}1285 \\
1368 \\
1223 \\
1303 \\
1392\end{array}$ & $\begin{array}{r}953 \\
990 \\
853 \\
1005 \\
1005\end{array}$ & $\begin{array}{l}16,500 \\
16,560 \\
15,306 \\
17,460 \\
16,620\end{array}$ \\
\hline \multicolumn{3}{|c|}{$\begin{array}{l}\text { Valor medio } \\
\text { Coef. de variación (\%) }\end{array}$} & $\begin{array}{r}1314 \\
5 \\
\end{array}$ & $\begin{array}{r}961 \\
7\end{array}$ & $\begin{array}{c}16,489 \\
5\end{array}$ \\
\hline $\begin{array}{l}\text { Madera } \\
\text { aserrada }\end{array}$ & $\begin{array}{l}1 \\
2 \\
3 \\
4 \\
5\end{array}$ & $180 \times 200$ & $\begin{array}{r}886 \\
851 \\
969 \\
946 \\
1064 \\
\end{array}$ & $\begin{array}{l}876 \\
800 \\
870 \\
836 \\
967\end{array}$ & $\begin{array}{l}16,860 \\
15,900 \\
16,260 \\
15,780 \\
16,980\end{array}$ \\
\hline \multicolumn{3}{|c|}{$\begin{array}{l}\text { Valor medio } \\
\text { Coef. de variación (\%) }\end{array}$} & $\begin{array}{r}943 \\
9\end{array}$ & $\begin{array}{r}870 \\
7\end{array}$ & $\begin{array}{c}16,356 \\
3\end{array}$ \\
\hline $\begin{array}{l}\text { Madera } \\
\text { antigua }\end{array}$ & $\begin{array}{l}1 \\
2 \\
3 \\
4 \\
5\end{array}$ & $\begin{array}{l}134 \times 145 \\
129 \times 157 \\
131 \times 142 \\
126 \times 146 \\
120 \times 129\end{array}$ & $\begin{array}{r}213 \\
272 \\
237 \\
283 \\
91\end{array}$ & $\begin{array}{l}208 \\
216 \\
218 \\
226 \\
191\end{array}$ & $\begin{array}{l}3,060 \\
4,860 \\
4,275 \\
4,725 \\
4,230\end{array}$ \\
\hline \multicolumn{3}{|c|}{$\begin{array}{l}\text { Valor medio } \\
\text { Coef. de variación (\%) }\end{array}$} & $\begin{array}{r}219 \\
35\end{array}$ & $\begin{array}{r}212 \\
6\end{array}$ & $\begin{array}{c}4,230 \\
17\end{array}$ \\
\hline
\end{tabular}




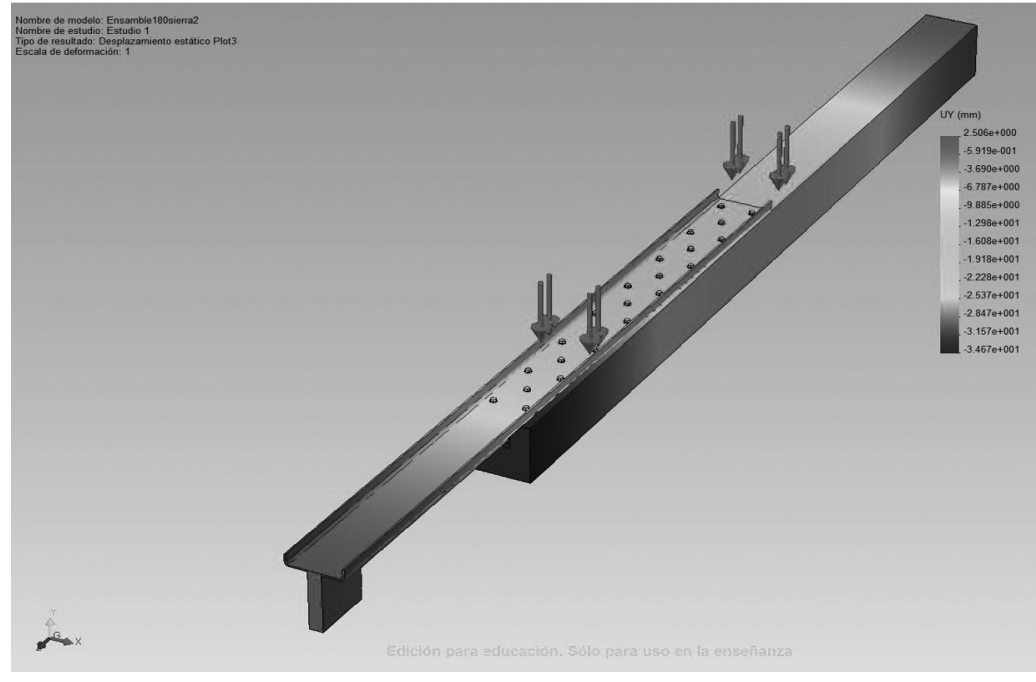

7. Modelo de la prótesis simulada en el programa CosmosWorks de cálculo por elementos finitos.
Con el fin de poder seguir trabajando en el diseño del sistema de refuerzo y consolidación una vez realizados los ensayos se procedió a establecer un modelo para su análisis por el método de los elementos finitos. Se utilizó el programa SolidWorks, ensamblando el refuerzo de acero y la madera en un fichero sobre el que se establecían las condiciones de contacto de acero-acero y acero-madera. Dentro de este estudio de contactos se incluye la disposición de tirafondos que unen los dos materiales para simular el ensayo real. El proceso de cálculo se realizó sobre el solver de Cosmos integrado en la plataforma CAD de SolidWorks, mallando los distintos componentes mediante tetraedros sólidos tridimensionales y refinando la trama de elementos en los distintos cambios de sección y espesores de las piezas.

En el mallado de sólidos se tuvo en cuenta cada material así como sus dimensiones. En el caso de las piezas de madera, se emplearon elementos de $32 \mathrm{~mm}$, mientras que en el acero se dispusieron elementos de $2 \mathrm{~mm}$ para poder alcanzar el mínimo de dos elementos en el espesor de la chapa. La configuración de cargas y apoyos en la pieza, siguiendo la UNE-EN 408, se establecieron permitiendo una distribución homogénea de las acciones a lo largo de la pieza, y de forma que el proceso real de ensayo quedara reflejado (Figura 7).

\section{RESULTADOS Y DISCUSIÓN}

En la Tabla 4, al comparar los valores de la rigidez a flexión inicial (de las piezas de madera completas) con la rigidez eficaz de las piezas con extensión metálica), se observa una disminución que es elevada para la madera laminada encolada $(961 / 1314=0,73)$ y poco marcada para la madera aserrada (870/943 = $0,92)$ y la madera antigua $(212 / 219=0,97)$.

El momento de inercia de los perfiles metálicos de refuerzo (con pletinas), $\mathrm{I}_{\mathrm{s}}$ es igual a $1.906 .385 \mathrm{~mm}^{4}$ para el perfil con dos pletinas inferiores y $460.848 \mathrm{~mm}^{4}$ para el perfil con una única pletina (Tabla 1). La rigidez a flexión de estos perfiles, considerando un módulo de elasticidad para el acero $E_{\mathrm{s}}=210.000 \mathrm{~N} / \mathrm{mm}^{2}$, es de 400 y $97 \mathrm{kN} \cdot \mathrm{m}^{2}$, respectivamente. Por tanto, los resultados antes expuestos sobre la reducción de la rigidez a flexión se explican fácilmente. El perfil de refuerzo supone un aumento de la rigidez en el tramo que acompaña a la pieza de madera. Sin embargo, en la zona de extensión la rigidez se limita a la del perfil de acero. El efecto global en el conjunto de la pieza implica una reducción que es más acusada cuanto mayor sea la rigidez de la madera, ya que la rigidez de la pieza metálica es la misma para madera laminada o aserrada.

Por otro lado, puede observarse una fuerte caída del momento máximo resistido comparando los valores medios del momento de rotura de las piezas de madera completas (Tabla 3) con los valores medios del momento de rotura de las piezas con extensión (Tabla 4). Se deducen las relaciones siguientes: 0,36 para la madera laminada encolada (16,489/46,334), 0,44 para la madera aserrada $(16,356 / 37,126)$ y 0,30 para la madera antigua $(4,230 / 14,194)$.

Esta pérdida de resistencia queda explicada fácilmente por el hecho de que el agotamiento del perfil metálico se alcanza antes que el agotamiento de la sección completa de madera. Si consideramos las secciones de acero de

Tabla 5

Comparación entre los momentos de rotura y los momentos de agotamiento por plastificación de los perfiles de acero

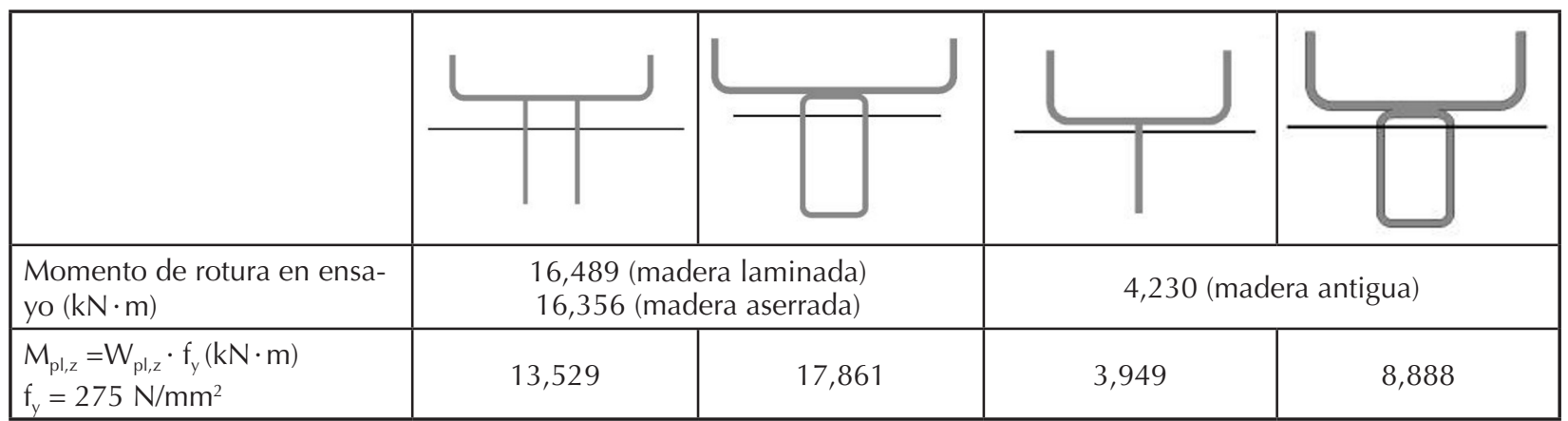


tipo 2 (plástica) según el Documento Básico de Seguridad Estructural de Acero del CTE (13) o el Eurocódigo 3 (14), y tomamos como límite elástico $275 \mathrm{~N} / \mathrm{mm}^{2}$, el momento de agotamiento por plastificación completa de la sección metálica alcanza los valores recogidos en la Tabla 5. Los momentos de rotura tienen valores que se encuentran muy cercanos a los que agotan los perfiles con pletinas.

De lo anterior se deduce que el perfil de refuerzo propuesto no permitirá salvar una zona degradada de la longitud ensayada $(1,14 \mathrm{~m}$ para una luz de $3,6 \mathrm{~m}$ o 0,86 para $2,7 \mathrm{~m})$, sino que deberá ser más reducida. Para estimar su posible alcance máximo en el caso de una viga biapoyada con carga uniforme se hace el siguiente razonamiento.

Sea una viga biapoyada de luz I y carga uniformemente distribuida q, (Figura 8). El momento en una sección a una distancia $x$ del apoyo viene dado por la siguiente expresión [1]

$$
M_{x}=\frac{q}{2} \cdot\left(l \cdot x-x^{2}\right)
$$

El momento máximo en el centro del vano, $M_{\max }$ es igual a q $\cdot 1^{2} / 8$; de donde se despeja el valor de q. [2]

$$
q=\frac{8 \cdot M_{\max }}{l^{2}}
$$

Sustituyendo el valor de q de la ecuación [2] en la ecuación [1], y llamando k a la relación entre el momento en la sección x y el momento máximo $\left(k=M_{x} / M_{\max }\right)$, se puede despejar el valor de la distancia $x$, que alcanza momento igual al de agotamiento del perfil de refuerzo

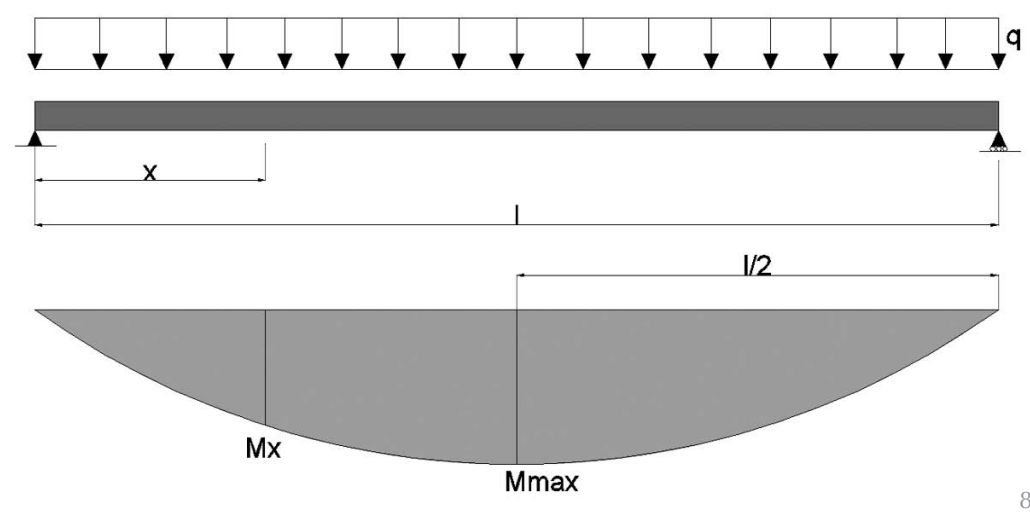

casos de las piezas de madera aserrada (180 $\times$ $200 \mathrm{~mm}$ ) con su perfil de refuerzo, y para las piezas de madera antigua $(130 \times 150 \mathrm{~mm}) \mathrm{con}$ su perfil de refuerzo más reducido.

Por ejemplo, para el caso de la madera antigua, donde $\mathrm{k}=0,30$, se obtiene un valor de $x=0,081 \cdot I$. Las vigas de madera antigua con un canto medio de $150 \mathrm{~mm}$ podrían salvar luces de unos $3 \mathrm{~m}$, por lo que la zona de extensión no debería superar 0,24 m. Por otro lado, normalmente, la carga máxima que puede soportar una viga de madera está limitada por el criterio de deformación, quedando el agotamiento por resistencia a índices del orden del $70 \%$. En este supuesto, el factor $\mathrm{k}$ pasaría a un valor mayor $(0,3 / 0,7=0,43)$, y el valor de $\mathrm{x}$ subiría hasta $0,37 \mathrm{~m}$.

En los ensayos se han observado dos modos de rotura. El primero ocurría en las piezas de madera laminada y aserrada donde el perfil metálico tiene dos pletinas en su parte inferior. El empalme de ambas pletinas con el perfil de tubo se realiza mediante cubrejuntas

\section{Tabla 6}

Distancias máximas de corte $\left(\mathrm{x}_{\max }\right)$ para las secciones de madera aserrada y antigua según el criterio de agotamiento (flexión o deformación)

\begin{tabular}{|c|c|c|c|c|}
\hline \multirow{2}{*}{$\begin{array}{c}\text { Sección } \\
(\mathrm{mm})\end{array}$} & \multicolumn{2}{|c|}{ Agotamiento por flexión } & \multicolumn{2}{c|}{ Agotamiento por deformación } \\
\cline { 2 - 5 } & $\mathrm{M}_{\mathrm{pl}, \mathrm{a}} / \mathrm{M}_{\max }$ & $\mathrm{x}_{\max }$ & $\mathrm{M}_{\mathrm{pl}, \mathrm{a}} /\left(\mathrm{M}_{\max } \cdot 0,7\right)$ & $\mathrm{x}_{\max }$ \\
\hline $180 \times 200$ & 0,44 & $0,081 \cdot \mathrm{I}$ & 0,63 & $0,195 \cdot \mathrm{I}$ \\
\hline $130 \times 150$ & 0,30 & $0,126 \cdot \mathrm{I}$ & 0,43 & $0,126 \cdot \mathrm{I}$ \\
\hline I: luz de la viga & \multicolumn{4}{|l}{} \\
\hline
\end{tabular}

bajo la carga q que agota a flexión la viga de madera. [3]

$$
x=l \cdot \frac{1-\sqrt{1-k}}{2}
$$

Esta será la distancia máxima que el perfil de refuerzo puede salvar en la zona de extensión, para no reducir la capacidad de carga de la viga. En la Tabla 6 se muestran los resultados para los soldadas. En este caso el fallo se alcanzaba por plastificación del acero del perfil de refuerzo. Se alcanza la carga máxima sin pandeo local de las alas comprimidas y se produce un giro de la sección. La soldadura de los cubrejuntas no rompe.

En la cara superior, las alas del perfil en $U$ sufrían una abolladura para permitir el giro por plastificación de la sección. La pieza de madera quedaba intacta después del ensayo; 


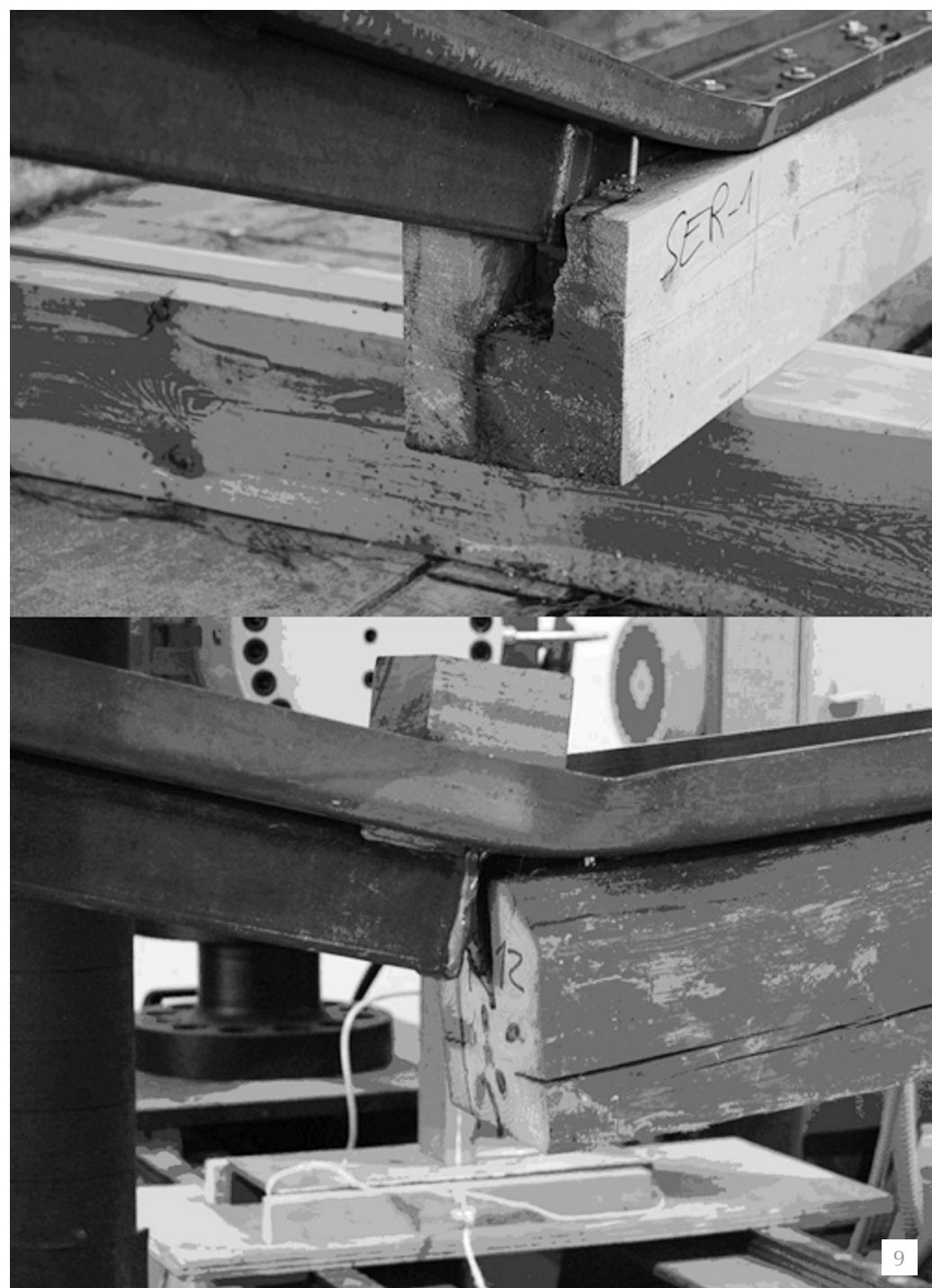

9. Zona de plastificación en la rotura; arriba en las piezas de $180 \times 200 \mathrm{~mm}$; abajo en las piezas de $130 \times 150 \mathrm{~mm}$. y los tirafondos que se encontraban más próximos a la transición entre la madera y el acero, algunas veces sufrían un efecto de extracción, cuando la plastificación se originaba ya dentro de la zona común con la madera.

El segundo modo de fallo se daba en las piezas de madera antigua donde el perfil de refuerzo únicamente tenía una pletina en su parte inferior. El empalme de esta pletina con el perfil del tubo estaba realizado con una chapa en la testa del tubo donde se soldaba la pletina. En este caso el fallo se alcanzaba de manera generalizada por la rotura de la soldadura, con un nivel de carga del mismo orden que el que produce la plastificación del perfil (Figura 9).

Para mejorar los resultados debería evitarse el punto débil que supone en el caso del perfil de una sola pletina, del empalme entre pletina y tubo. Para ello podría plantearse una unión con mayor capacidad sin que quede limitada por la soldadura. Dado que el fallo, tanto en perfiles de una como de dos pletinas, se alcanza con valores del momento del orden del que produce la plastificación, también sería deseable emplear un perfil más resistente. Por ejemplo, podría prolongarse el tubo dentro de la pieza de madera una longitud suficiente como para llegar a haber transmitido gran parte del momento al a propia madera. En cualquier caso también se puede mejorar el resultado utilizando un acero de mayor límite elástico como el S355.

\section{CONCLUSIONES}

El perfil metálico de refuerzo estudiado permite un aumento de la rigidez cuando se extiende en prácticamente toda la longitud de la pieza. Sin embargo, su utilización como elemento de sustitución de la madera en un tramo supuestamente degradado cercano al apoyo reduce este efecto de incremento de rigidez. Si sólo se utiliza como consolidación de apoyo, con las condiciones estudiadas, provoca únicamente una ligera pérdida de rigidez no relevante.

Las soluciones ensayadas en este trabajo alcanzan su agotamiento por una plastificación del perfil de acero bajo momentos máximos inferiores a la capacidad de la viga de madera por sí sola. Para no reducir su capacidad portante será necesario limitar la longitud de extensión (zona degradada de la cabeza de madera) a un valor que en la práctica puede estar entre el 8 y el $17 \%$ de la luz de la viga, aproximadamente.

Para mejorar su comportamiento se sugiere la utilización de un acero de calidad S355 así como la posibilidad de emplear una sección de refuerzo mayor que permita aumentar su capacidad de flexión.

El número de ensayos realizado en este trabajo es reducido y por tanto las conclusiones que se obtienen deben considerarse como un primer paso para una mayor profundización mediante trabajos posteriores en este campo. No obstante, la simulación realizada mediante el método de los elementos finitos ha demostrado grandes posibilidades como método de diseño para la mejora de las soluciones propuestas.

\section{AGRADECIMIENTOS}

Laboratorio de Estructuras de Madera del CIFOR-INIA, Madrid. 


\section{BIBLIOGRAFÍA}

(1) F. Arriaga; F. Peraza; M. Esteban; I. Bobadilla; F. García: Intervención en estructuras de madera. p. 476., Editorial AITIM, Madrid, 2002.

(2) Landa, M.: "Nuevas técnicas de reparación de estructuras de madera. Elementos flexionados. Aporte de madera-Unión encolada I. Metodología de puesta en obra". Revista de Edificación no 28, Pamplona (1999), pp. 32-38.

(3) Landa, M.: "Nuevas técnicas de reparación de estructuras de madera. Elementos flexionados. Aporte de madera-Unión encolada II. Metodología de puesta en obra". Revista de Edificación n 29, Pamplona (1999), pp. 30-36.

(4) Anon: "The age of resin". The Architects Journal, Feb. (1982) pp. 56-58.

(5) Mettem, C. J.; Page, A. V.; Robinson, G. C.: "Repair of structural timbers. Part I: Test on experimental beam repairs". TRADA, Technical Report. Reino Unido (1993).

(6) González-Bravo, C.: "Recuperación de la capacidad mecánica en piezas de madera solicitadas a flexión en estructuras tradicionales operando por la cara superior mediante refuerzos y prótesis metálicas". Tesis Doctoral. Universidad Politécnica de Madrid, ETS de Arquitectura. 265 p. (2007) PDF file: http://oa.upm.es/442.

(7) González-Bravo, C.; Arriaga, F.; Díez, R.: "Bending reinforcement of wooden beams with steel cross sections". Proceedings of the 10th World Conference on Timber Engineering. Miyazaki, Japan (2008).

(8) González-Bravo, C.; Arriaga, F.; Maldonado, L.; Díez, R.: "Refuerzo de vigas de madera mediante perfiles metálicos situados en la cara superior". Mater.Construcc.. Vol. 60, n 298 (2010) pp. 123-135. doi: 10.3989/mc.2010.47408.

(9) UNE-EN 10025-1:2006. Productos laminados en caliente de aceros para estructuras. Parte 1: Condiciones técnicas generales de suministro. (Hot rolled products of structural steels - Part 1: General technical delivery conditions).

(10) DIN 571 Tirafondos para madera con cabeza hexagonal (Hexagon head wood screws).

(11) UNE-EN 408:2004. Estructuras de madera. Madera aserrada y madera laminada encolada para uso estructural. Determinación de algunas propiedades físicas y mecánicas. (Timber structures. Sawn timber and glued laminated timber for structural use. Determination of some physical and mechanical properties).

(12) EN 13183-2:2002. Contenido de humedad de una pieza de madera aserrada. Parte 2: Estimación mediante el método de la resistencia eléctrica. (Moisture content of a piece of sawn timber. Part 2: Estimation by electrical resistance method).

(13) Documento Básico de Seguridad Estructural, Acero. Código Técnico de la Edificación. Ministerio de la Vivienda. (BOE 23/102007 y 25/01/2008).

(14) UNE-EN 1993-1-1:2008. Eurocódigo 3: Proyecto de estructuras de acero. Parte 1-1: Reglas generales y reglas para edificios. 